\title{
Bas a \\ Finding Facts vs. Browsing Knowledge in Hypertext Systems
}

\author{
Gary Marchionini and Ben Shneiderman \\ University of Maryland
}

\begin{abstract}
$\mathbf{F}$ or hypertext and electronic information systems to be effective, designers must understand how users find specific facts, locate fragments of text that satisfy information queries, or just browse. Users' information retrieval depends on the cognitive representation (mental model) of a system's features, which is largely determined by the conceptual model designers provide through the human-computer interface. Other determinants of successful retrieval include the users' knowledge of the task domain, information-seeking experience, and physical setting.

In this article we present a user-centered framework for information-seeking that has been used in evaluating two hypertext systems. We then apply the framework to key design issues related to information retrieval in hypertext systems.
\end{abstract}

\section{Hypertext}

Hypertext and other electronic information systems overcome human limitations by providing mechanisms for compact storage and rapid retrieval of enormous volumes of textual, numeric, and visual data. The importance of these systems lies in their potential capacity to augment and amplify human intellect. We need this capacity because of the exponential

\begin{tabular}{l}
\hline \\
Hypertext systems \\
will change the way \\
people read and write. \\
Two evolving systems, \\
Hyperties and the \\
Electronic \\
Encyclopedia, \\
demonstrate the \\
possibilities and \\
problems.
\end{tabular}

growth, increasing complexity, and multidisciplinary nature of scientific, economic, medical, and other knowledge.

Early information retrieval systems searched large databases of records (library card catalogs, legal citations, scientific journal abstracts, etc.) to retrieve items that satisfied a Boolean, keywordoriented query. A second strategy made information resources available in videotex systems through menu selection hierarchies. Large full-text databases were explored with string search strategies to locate lines or paragraphs with desired patterns. A fourth familiar strategydatabase management systems-retrieved structured records of accounting, scientific, or other data according to the search logic of a procedural program or a precise query language.

The applicability of these strategies overlap, but the differences reveal the diverse approaches and tools that exist. A physician trying to find every clinical study of Parkinson's disease is very different from a high school student needing information for a term paper.

A new approach-hypertext-has recently joined electronic information systems. The term, coined by Ted Nelson, ${ }^{1}$ describes a vast network of text fragments linked together, an electronic writing and reading system that uses the power of the computer for more than editing and display. Nelson's followers worked on a prototype and Douglas Engelbart ${ }^{2}$ created a variant approach during the 1960s. But only in the past few years have practical, commercially viable systems and provocative research implementations appeared. (For a review, see Conklin. ${ }^{3}$ For discussions of particular systems, see Yankelovich, Meyrowitz, and van Dam, ${ }^{4}$ 
Halasz, Moran, and Trigg, ${ }^{5}$ and Goodman. ${ }^{6}$ )

Hypermedia and hypertext systems allow users to traverse complex networks of information quickly. Authors can easily link passages and references and collapse or expand outlines; readers can freely move among text fragments to find sources of quotations, journal article references, definitions, and related passages.

From the writer's point of view, hypertext systems are the next generation of word processing. In addition to word processing features like block moves, search and replace, and spell or style checking, hypertext writing tools may support and extend the writing process with telescoping outlines, posted notes that do not affect the main text, electronic bookmarks, and browsing modes.

From the reader's point of view, hypertext systems are a new generation of database management. Full text is accessible from multiple perspectives, for various purposes, and through different search strategies. Thus, hypertext databases are more malleable to the user than print or early electronic text formats.

In this article we focus on hypertext from the reader's perspective, in particular, how users find information in such systems.

Hypertext usage depends on what mental models users have for the system. These mental models in turn depend on the conceptual models used by designers to create and present the system. Therefore, effective use depends on better understanding of how information-seeking processes are learned and applied.

Since hypertext systems have a brief history of application, we have sparse evidence for their effectiveness, let alone proven principles to guide design. Advocates enthusiastically point out the similarity between human associative memory and the network of text fragments that allows freedom in linking ideas. While there are undoubtedly information search tasks that hypertext suits, promoters may fail to realize that the very same freedom of linkage they admire can complicate some search or learning tasks.

Present systems may support browsing strategies attractive to end users but inefficient for fact retrieval. To compensate, cumbersome analytical strategies that take advantage of indexing to improve retrieval may be supported; however, the overall design may become complex. Analytical strategies include consulting thesauri before search, using Boolean connectives, and systematically iterating queries.

Determining criteria for optimal mixes of browse and analytical support is critical to development. Balancing the power for retrieval with the ease of understanding is a central problem for designers of future systems. We believe that the solution is to provide flexible, powerful human-computer interfaces to maximize benefits for every community of users.

\section{Three pillars of hypertext research}

The maturation of software and hardware and the widespread availability of personal and mainframe computers have stimulated great interest in the design of electronic information systems and made possible search strategies impractical in manual systems. Research related to human performance with hypertext and other electronic information systems integrates methods and ideas from information retrieval, interface design, and cognitive science.

Information retrieval. Research related to on-line searching has focused on systems that aid professional intermediaries in finding a small number of "hits" in a large collection of records (library card catalogs, scientific journal abstracts, UPI reports, etc.). The emphasis has been on designing systems that aid or replace professional intermediaries (see Marcus ${ }^{7}$ for an example of an actual system).

Professional on-line searchers primarily clarify information requests and retrieve relevant information for end users. They carefully plan in advance, consult thesauri, and combine terms in systematic and precise steps by applying logical connectives (AND, OR, NOT) and by adjusting proximity limits (the range of words within which query terms must cooccur) and scoping limits (the range of documents over which search takes place). Unless they are themselves a part of a research team effort, they act as communication channels, locating and transferring information to end users who interpret and apply it.

The primary goal of on-line searchers is to retrieve and communicate information efficiently - their analysis usually focuses on the facets of a request for information, not on the problem that motivated the question or the possible application of the answer. Search intermediaries rarely browse informally, because focusing on the goal yields ef ficient and cost-effective performance. Their analytical strategies include much preplanning, application of Boolean connectives, and systematic iterations of the querying and refinement process.

End users, on the other hand, often browse despite accruing costs because they have long-term commitments to an area of research and may later benefit from extraneous information in that area. In other words, end users rationalize inefficient information-seeking strategies by hoping that incidental learning will have a beneficial cumulative effect. Browsing is an exploratory, information-seeking strategy that depends on serendipity. It is especially appropriate for ill-defined problems and for exploring new task domains.

Today's electronic retrieval systems were designed for use by professional intermediaries, or to emulate their performance. These systems focus on coding, indexing, and cross-referencing (organization for retrieval) rather than on meaning, readability, and assimilation (organization for understanding). Systems meant for end users must take into account these differences and support appropriate information-seeking strategies.

Hypertext systems differ from existing on-line retrieval systems in that they encourage informal, personalized, content-oriented information-seeking strategies. Hypertext system users can actually apply information during the retrieval process by noting context, and during browsing by saving, linking, or transferring text or images. Of particular interest in our research is the support of end users through flexible and powerful human-computer interfaces that balance end-user browsing strategies with efficient analytical strategies like those used by professional intermediaries.

Interface design. Improvements in human-computer interface design have come rapidly in the last decade. Hardware advances offer designers a range of input devices (such as mouse or touch panel) and output devices (such as high-resolution screens). Likewise, software advances allow designers to choose from a variety of selection mechanisms in addition to traditional command languages. (See Shneiderman $^{8}$ for a set of interface design principles and references to the growing body of literature.) Although command languages offer expressive power to expert users, a variety of menu selection styles 


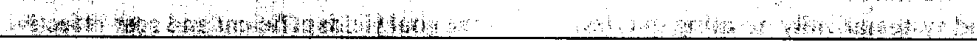

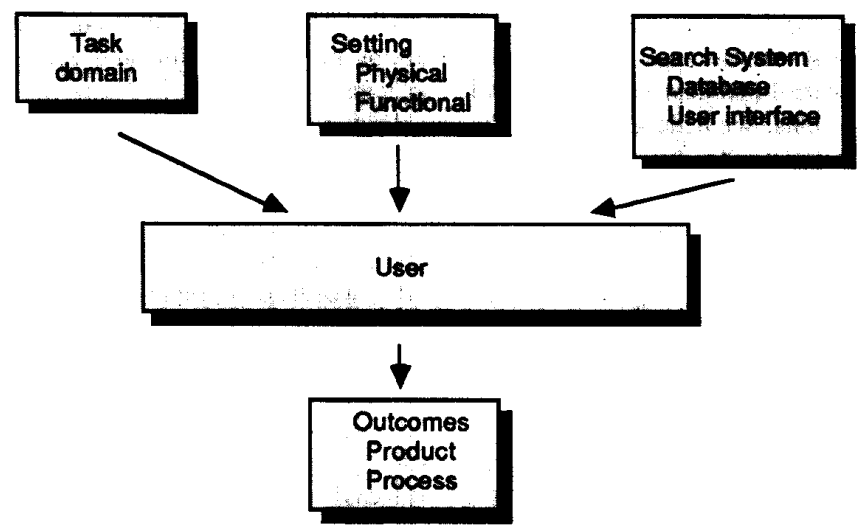

Figure 1. This information-seeking framework helps to identify the determinants of success. Issues include the complexity of the task domain, the physical and functional setting, the search system structure and user interface, and the user's knowledge of each. The outcomes of a search are specific information and the sequence of steps to generate the product.

(pull-down, icons, embedded) offer novice and casual users ease of use. Powerful personal computers have now made visually oriented direct manipulation styles possible. Instead of remembering commands or traversing menus, the user sees a representation of the "world of action." Because the user points (with mouse, touchscreen, etc.) at given objects and actions, the impact of actions is immediately visible, thereby reducing errors and speeding performance. Actions should be rapid, incremental, and reversible to promote a sense of mastery, control, and confidence. Examples include display editors the Macintosh or Star desktop, most video games, and many CAD/CAM systems.

Direct manipulation interfaces may consume more system resources, may be more difficult to implement, and may not always be as efficient to use as other interfaces, but in general they lead to less cognitive load in using computers, thus enabling users to apply severely limited human working memory to the task domain. As with information retrieval, a key design problem is balancing the ease of learning afforded by direct manipulation and access to powerful features for experts.
Cognitive science. The linchpin of an information-seeking theory is the human user. A first step to understanding information-seeking in electronic environments is to develop an understanding of the basic cognitive processes that guide information-seeking. We lack a clear definition, much less understanding, of the interactions among an information seeker's knowledge about a problem, past experience in searching for information, and knowledge of possible sources for information. Furthermore, since any system that supports information-seeking must structure knowledge to make it accessible, the systems themselves affect how users think when using them. The emerging interactive systems that encourage dia$\log$ and progressive query formulation instead of requiring direct commands can serve as environments for testing cognitive augmentation.

Cognitive scientists have proposed dynamic internal representations-mental models - that can serve as the focal point for building and testing a theory of information-seeking. (See Gentner and Stevens $^{9}$ for a set of examples, and Borgman $^{10}$ for an example applied to information-seeking.) A mental model is a cognitive representation of a problem situation or system that is active in the sense that it can take inputs from the external world and return predictions of effects for those inputs. It can thus be "run" internally and the results used to make decisions about actions. Mental models allow us to both understand problem situations and predict consequences of actions contemplated for solving problems. Users develop mental models for systems through reading documentation, training, experience with systems, and comparing them with previously encountered systems.

The rules for navigating a database as well as the mechanisms for interaction (input and output) affect the user's scope of application-determine what the user thinks is possible with the system. Designers must not only consider how to structure knowledge from a system performance vantage, but also consider what views and correspcnding navigational tools are provided for the user. The views and navigational tools will be easily assimilated into a mental model for a system if they are familiar. This reasoning lies behind the desktop and other metaphors. However, a tension exists between the learnability and applicability of a system; a too-rigid metaphor may limit the development of users' mental models for new systems. Designers must know how users seek information in traditional print systems and existing electronic systems if they are to produce effective interfaces for new systems. An understanding of how users learn and apply existing systems will allow designers to build interfaces that accentuate the familiar and serve as bridges to the new features and functions of hypertext systems.

\section{A framework for information-seeking}

The following framework for information-seeking is meant to guide designers of hypertext systems and users who apply them to write and read hypertext documents. Figure 1 presents an overview of these components and their relationships. The interactions of these components determine the overall performance of an information-seeking system.

Setting. The setting within which information-seeking takes place constrains the search process. The physical setting (in a user's private office versus in a public 
place with a line of impatient would-be users nearby) determines physical constraints such as the amount of time allocated, physical accessibility, and cost. These act as external control mechanisms for the search process.

The setting also determines functional constraints such as the motivation and purpose for conducting the search, whether pleasure, job assignment, or ongoing research interest. The setting actually enables the search task. Thus, the setting helps delimit the task domain and motivate the user, and affects the selection and application of the search system.

Task domain. A task domain is a body of knowledge, whether hematology or bridge design, composed of entities and relationships. Task domains vary in complexity (number of entities and relationships), specificity (similarity of the entities and relationships), and evolutionary status (clarity of definition of the entities and relationships, and their rate of growth and change). These characteristics determine the amount of information and level of organization for a task domain.

The amount of information and level of organization vary immensely across task domains. The task domain is critical because it affects the strategies and search systems available. A task domain like hematology offers substantial on-line information from various vendors in various forms, from abstracts to full texts. On the other hand, a task domain like contemporary music offers little on-line information and limited access through such common entry points as subject headings. Furthermore, the type of search system available depends on the task domain. For example, in the humanities the primary vehicle of information is the book, whereas in the physical sciences technical reports and journal articles dominate.

Search system. The search system consists of a database and a human-computer interface that allows access and manipulation of the database through a set of search rules. A print encyclopedia consists of words on pages and alphabetical ordering rules for using the index or finding articles by titles. In the case of electronic search systems, some of the rules and structures are embodied in software.

The user's first concern about the database is content-whether it is primary or secondary (pointer) information, full-text or symbolic, and how it matches the task domain and the information problem at hand. Once the user has selected the right database for the task, the organizational structure of the database becomes the primary determinant of information-seeking performance.

The database may be a simple sequence of full-text passages, a set of fixed-length records related through hash coding or $b$ trees, or a loose web of graphics and text linked through pointers. The organizational scheme chosen by the designer is critical to performance and will influence the interface as well. In turn, physical database organization is influenced by hardware and media. (See Zoellick ${ }^{11}$ for a discussion of how CD-ROM characteristics affect design.)

The human-computer interface is a communication channel with hardware and software components. The physical input/output devices, selection and feedback mechanisms, and search features determine the power and flexibility of a search system. The search system works because the designer has a view of the typical user when creating the interface. Primitive systems have static internal representations for users; they are controlled by the user and a set of default conditions that reflect the system designer's conceptual view of typical users and their information problems. Information systems that have some adaptive capability enable users to change aspects of the internal representations or the user interface.

The search system is critical because it structures knowledge and defines how it is accessed. The way knowledge is organized and made available affects the strategies used to access this knowledge and thus information-seeking performance.

User. Each user is unique, possessing mental representations for task domains. A generic knowledge base of informationseeking experiences includes mental models for various search strategies, dynamic mental models for search systems, and a control mechanism for relating these internal representations to one another and to external entities. Of particular interest for designers is how users develop mental models for new systems and how they apply these mental models when using systems.

A user's mental model for a search system is critical to the search process because it determines expectations for outcomes and the search strategies used. A mental model is active-it "runs" internally before action takes place. A user's mental model includes both the entities and rela- tionships represented (how knowledge is structured) in the search system and rules for controlling the system.

Users can be classified along three continua: frequency of use, complexity of application, and general range of computer experience. The position of users in a space defined by these dimensions determines how quickly and accurately they will develop a mental model for a system and how effectively they can apply it.

Low-frequency users may develop accurate mental models for what the system can do, but forget the details of system use. These users need menus and on-line reference aides. Frequent users, on the other hand, may prefer commands to expedite their use of the system.

Users who perform only straightforward tasks do not need all the features a system supports and thus need only abbreviated menus. On the other hand, users who push the limits of a system access the complete hierarchy of menus, invoking every system feature.

Users with little computer experience have more to learn and fewer mental models of related systems from which to draw analogies. These users depend more heavily on experience with the system to develop their mental models. Their initial experiences must be simple enough to allow success and continued learning. On the other hand, just as first impressions are socially critical for future interaction among people, initial experiences with a computer system play a critical role in framing a person's emerging mental model for a system. If the initial experiences lead to ambiguous or inaccurate mental models, users will have difficulty extending their use of the system.

Because users vary so much in their individual abilities, experiences, and purposes, designers must struggle with providing an interface that does not frustrate or confuse yet is rich enough to support the eventual appendage of a full set of system features.

Outcomes. Outcomes for informationseeking include both products and a process. Products of search, from individual facts to complete documents that are interpreted to satisfy the problem condition, provide one basis for evaluating search effectiveness. Typical measures of search products include assessment of relevance or utility by users during or after search, structured or informal subjective evaluations, and examination of the resultant products or artifacts (for example, documents or abstracts). 


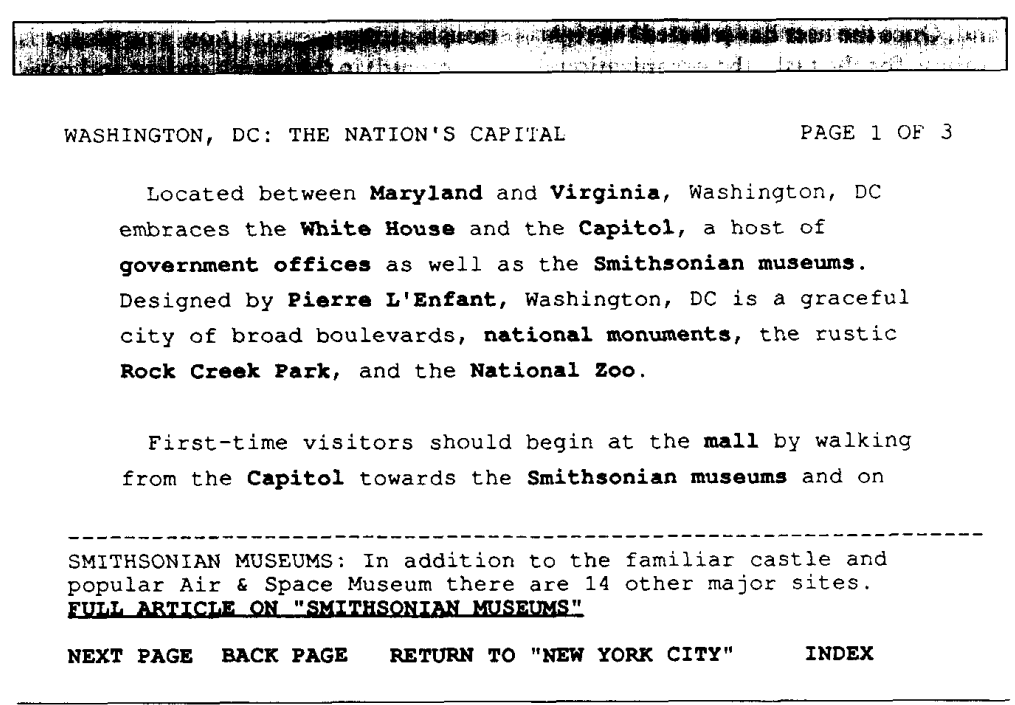

Figure 2. This Hyperties display on an IBM Personal Computer shows the highlighted embedded menu items available for selection by touchscreen or arrow keys. The user can follow a topic of interest, turn pages (NEXT or BACK), RETURN to the previous article, or select the INDEX.

The behavioral moves made by users and systems during a search - the search process-also help in evaluating performance. Evaluators assume that user behaviors are manifestations of internal information-seeking strategies, which are themselves "runs" of the user's mental model for the search system.

Although information units retrieved by the system are easily collected for analysis, the analysis of the search process causes more problems. Examination of paths taken and decisions made in jumping to other nodes allow us to make inferences about users' cognitive activity and provide evaluative data on system effectiveness.

Another important aspect of the search process is that the experience itself becomes part of the user's knowledge for dealing with future information problems. Therefore, consistency in design can help support incremental development of users' mental models.

\section{Hypertext systems research}

Since the information-seeking components described above are multifaceted variables, research efforts that attend to their interaction must be longitudinal and cumulative. We have studied two hypertext systems extensively and describe some of our results to encourage related empirical work and raise design issues.

Both these systems are what Conklin ${ }^{3}$ calls structured browsing systems. The databases used were static, in that users could not change them. ${ }^{*}$ Both systems were designed for casual or novice users and thus the research efforts reported here focus on interface issues and users' mental models. In most of the work reported, the researchers controlled setting and task domain to focus on the user and system components of information-seeking. This research is further limited to information retrieval; the results and design issues discussed are thus directed to the reading aspects of hypertext systems.

Hyperties. Hyperties (hypertext based on the Interactive Encyclopedia System) enables users to easily traverse a database of articles and pictures by merely pointing at highlighted words or images in context (see Figure 2). Highlighted or colored words or phrases within the text become the menu items, selectable using a point-

${ }^{*}$ Hyperties allows both writing and reading, but in sep arate modes, called Author and Browser. ing device. Rather than isolated and explicit menu items, the context of embedded menu items provides information and cues for the selection of further information.

This embedded-menus approach and the simple user interface enable users to explore large databases easily. Hyperties has been used to create a guide to the Student Union, an on-line help service for a bibliographic search system, training materials about software, a supplement for a museum exhibit about photographer David Seymour, an encyclopedia about the Holocaust, an introduction to the computer science department, and so forth.

Hyperties users merely touch or use a cursor to specify topics of interest; a brief definition appears at the bottom of the screen. Users may continue reading or ask for details about the selected topic. An article about a topic may be one or more screens long and contain several pictures. As users traverse articles, Hyperties traces the path and allows them to return to previous articles, all the way back to the introductory article. Users can also select articles and pictures from an index.

Hyperties has been under development since 1983 at the Human-Computer Interaction Laboratory at the University of Maryland. ${ }^{* *}$ In addition to the standard IBM PC version, there is a Sun workstation Hyperties browser that provides two windows with 34 lines in each and graphics with embedded menus (see Figure 3 ).

Embedded menus are good examples of specialized indexing for systems that emphasize understanding rather than retrieval. As local indexes they highlight semantic relationships rather than physical relationships. Questions about how deep embedded menus should be, whether they should be available in definitions as well as articles, whether networks are superior to hierarchical structures, whether string searching should be supported, and how alphabetical indexes and embedded menus complement or interfere with one another are under investigation.

It is clear from the information-seeking framework that the type of database, user, and task affect the use of embedded menus. Results of an experiment comparing versions of Hyperties with and without embedded menus demonstrate the critical role of tasks and users' existing mental **Hyperties was first implemented in APL by Dan
Ostroff, and has been rewritten in C twice. It is disOstroff, and has been rewritten in C twice. It is dis-
tributed by Cognetics Corporation of Princeton Junction, N.J. 


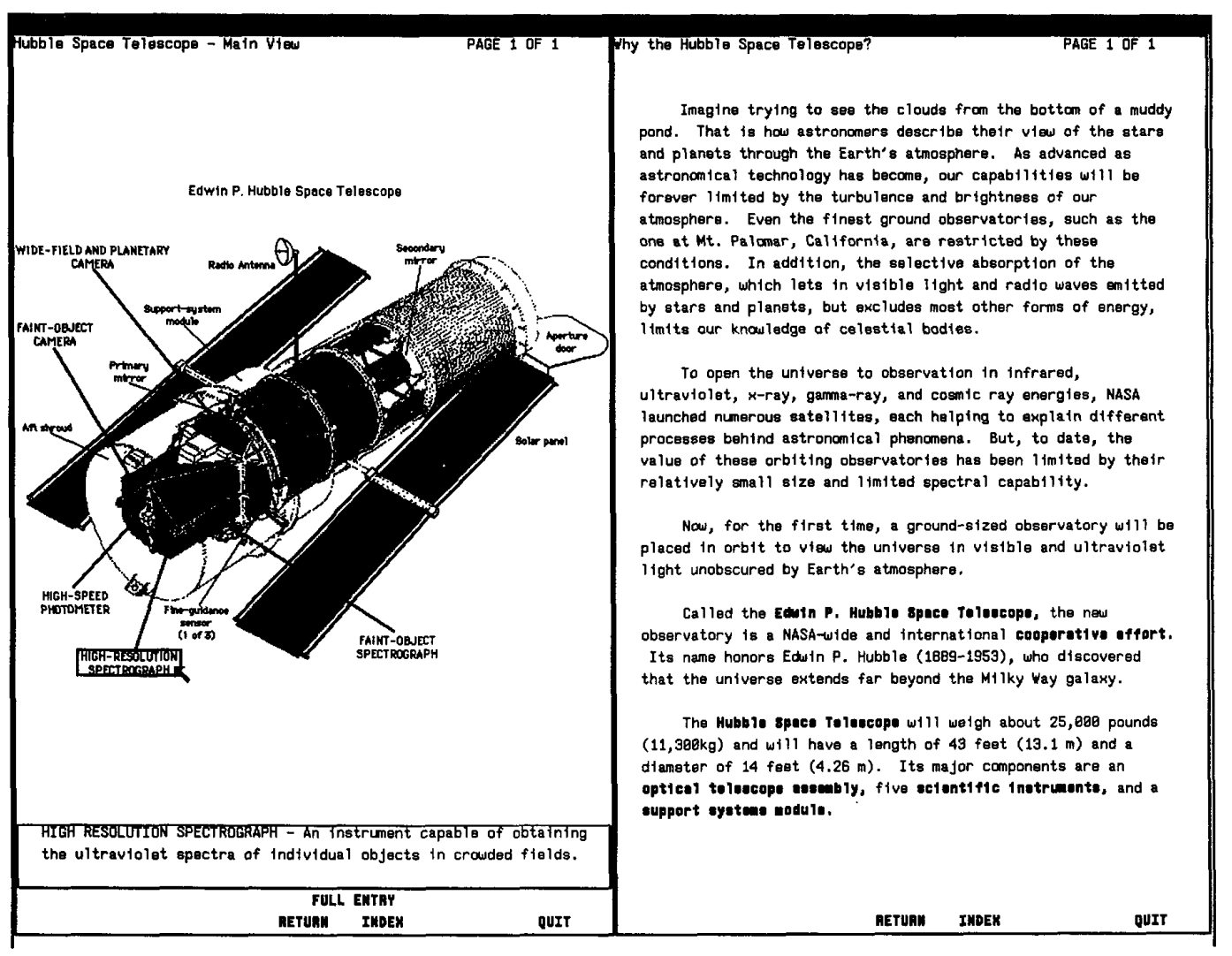

Figure 3. This Hyperties display on a Sun 3 workstation demonstrates the advantages of a larger screen, two windows, and touchable graphics. With the mouse users can select highlighted phrases in text or point at components of the space telescope to retrieve more information. The components pop out and the user can elect to see a diagram of the contents.

models for search tasks. When subjects were asked to perform efficiently in searching for specific factual information in a Hyperties database, the predominant strategy ( 14 of 16 subjects) was to use the alphabetical index. These subjects, experts at searching in manual indexes, used the familiar strategy rather than a strategy the new system was designed to promote.

In contrast, a log of usage in two museums over eight months showed that more than two-thirds of all selections were through the embedded menus, thereby demonstrating the orientation toward browsing in a museum setting.

January 1988
Another study required subjects to use embedded menus or the index to search for specific facts in the same database. All tasks could be accomplished with either search strategy, but index users located facts more quickly than embedded menu users. The differences in performance between the groups became progressively smaller as more searches were conducted. This result suggests a learning effect as subjects become more familiar with the conceptual aspects of embedded menus. By systematically varying task and user variables we expect to build guidelines for future implementations that support a range of information-seeking activities ranging from fact retrieval to general browsing.

Another research study with Hyperties $^{12}$ demonstrated the advantage of embedded menus versus explicit menus in speed of fact retrieval and subjective preference. Two experiments explored several pointing devices and demonstrated the speed advantage of the jump-arrow method over the mouse, probably because of novice users overshooting or undershooting the highlighted word or phrase with the mouse. The arrow keys forced easily controlled discrete moves. A touch- 
screen proved still faster and yielded higher satisfaction, although the touchscreen used produced high error rates. We have now developed touchscreen strategies that are fast and also accurate enough to point at single characters. In one implementation, the user touches the surface to produce a cursor (above the finger) that can be moved rapidly onto the desired target. When satisfied that the selection is correct, the user removes the finger to initiate action.

Of particular interest are our studies of Hyperties versus paper versions of the same database. Previous studies have shown a 30-percent penalty in reading speed for typical computer displays versus typewritten text, and up to a twofold disadvantage for computer users performing certain fact retrieval tasks. ${ }^{8}$

Larry Koved at the University of Maryland used the Hyperties approach for two experiments with on-line maintenance manuals for electronic equipment. A treestructured and a linear form of a 52-page maintenance manual were prepared for screen presentation and in paper form. Differences in the time taken to accomplish 12 tasks showed use of the paper versions to be significantly faster. No significant differences for speed or error rates showed up between the tree and linear versions. However, a pruning algorithm applied to the electronic version to trim text unrelated to the task cut the time in half, suggesting an advantage in the flexibility of electronic information systems.

In a recent study using a larger database (106 articles of 50 to 2000 words) on Austria and the Holocaust, paper versions with an index had a speed advantage with simple fact retrieval questions. But as the query complexity increased, the Hyperties users performed equally rapidly. Moreover, users dramatically preferred Hyperties over paper.

Hyperties was designed to support easy browsing of text and graphic databases. Results of many evaluative studies demonstrate that even novices find it easy and effective to use.

Electronic Encyclopedia. Another system we examined is the full text of Grolier's Electronic Encyclopedia on CDROM (see Figure 4). The print version of the encyclopedia occupies 20 volumes. The hypertext version consists of 60 megabytes of text and 50 megabytes of indexes that contain pointers to each occurrence of every word in the encyclopedia, all occupy- ing less than one-fifth of a single CD-ROM disc. The powerful search software for this system provides rapid access to all occurrences of any word or phrase entered by the user. The Boolean connectives AND, $\mathrm{OR}$, and NOT are supported as well as right truncation, character masking, proximity limitations from one to 999 words, and scope limitations for various sections of articles.

One study ${ }^{13}$ examined the informationseeking strategies used by elementary school students. Results demonstrated the tendency of novices to use low cognitive load browsing strategies. The children used the system successfully even though their application of Boolean connectives was weak or incorrect. Their success came from the system itself; results of queries were displayed as alphabetical lists of titles with frequency of term occurrence. A single keystroke would then retrieve a full article with the query terms highlighted. This interface facilitated a scan and select strategy. Searchers simply entered a query scanned the resulting list of articles for titles that were semantically relevant or had high frequency of term occurrence and then selected the full text of the article and scanned for term occurrences (highlighted in the text) to locate relevant sections. Thus, the hypertext features of the search system compensated for some of the formal search inadequacies of these children.

Follow-up studies (available from the authors) were conducted with high school students to compare print and electronic searches and examine the development of mental models for this system. One study examined the default conditions provided by the search software. The results indicate that setting proximity defaults to a paragraph seem optimal for a generic encyclopedia. The default scope condition on searching all categories of the article also proved optimal for this database. These results may not extend to other databases, since the theoretical framework predicts that strategy depends partly on task domain, and some domains may require special default settings. For example, technical documents-typically terse and specific-may require proximity settings that span more than a paragraph.

Another study simulated the effects of adding a controlled vocabulary to the search system. Many novices cannot articulate morphological variations or synonyms for search terms. Three levels of vocabulary control were tested: morphological (using variant forms and endings for terms); synonymic (using synonyms for terms); and semantic (using a hierarchical thesaurus that included broader, narrower, and related terms). Subject searches were reconstructed with these controls applied and analyzed for recall (ratio of relevant items retrieved to total relevant items in the database) and precision (ratio of relevant items retrieved to total number of items retrieved). All levels of control improved recall, but only semantic control improved precision, albeit only slightly. Designers of hypertext systems must decide whether to support vocabulary control of any sort, and then at what levels.

The power of this search system was demonstrated in another study that compared two groups of novice users trained to use different search strategies. One group learned to use a scan and select strategy and the other learned an analytical search strategy (using Boolean connectives and planning complete queries in advance). Subjects who used the analytical strategy performed slightly more efficient searches with respect to time and number of keystrokes, but exhibited no significant differences in effectiveness (success in finding information and quality of essays produced).

Overall, the experiments conducted with this system suggest that novices can successfully apply hypertext by using low cognitive load strategies modeled on existing browsing strategies, and that the power of the system overcomes some of their search inadequacies. However, these successes were accomplished using minimal capabilities of the system (default settings) for tasks appropriate to the users and the database.

Some anticipated problems of menu floundering and disorientation did arise. For example, after finishing with an article many subjects moved back up the menu hierarchy to the query formulation screen and entered the same query again (requiring another CD-ROM access) rather than moving up a single level to the screen of article titles already retrieved for that query. Another common move was to use the backspace key to erase a query rather than using the single keystroke for erasing a query. These subjects also became disoriented within an article because of the way the system put sections of text on the screen. When a user selects an article from the title list, the text of the article is displayed beginning with the first paragraph that contains the query term(s). Many students did not notice that they were in the 


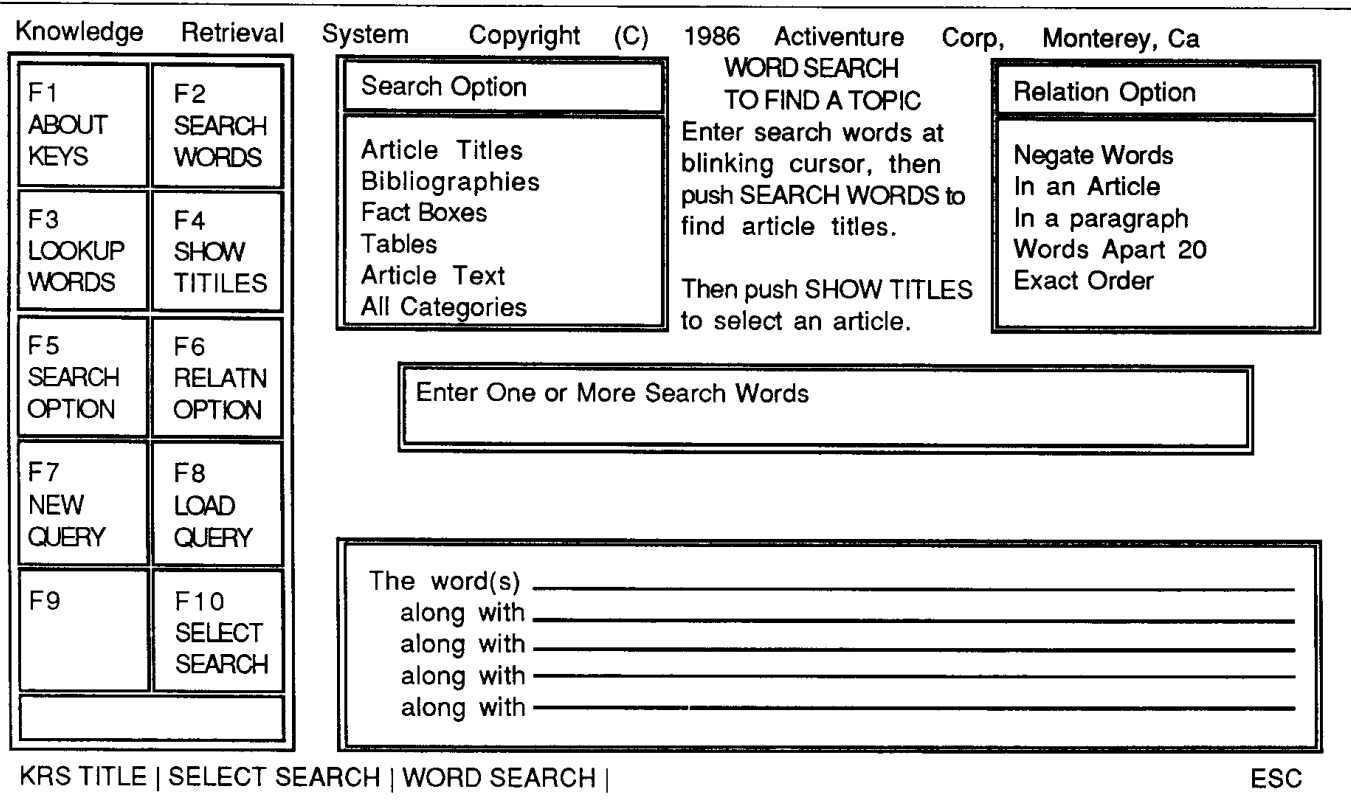

Figure 4. This main menu from Grolier's Electronic Encyclopedia offers users several search options and information about function key usage.

middle of an article rather than at the beginning.

A similar problem came up with subjects who could not distinguish when they had the beginning or end of an article on the screen. Since the system provided no explicit positional cues, they erroneously continued to page up or down when at the beginning or end, respectively.

In general, the interface actually provided no feedback for queries that yielded no results. It also presented complex and dense screen displays (see Figure 4). We can easily overcome these problems with closer attention to the results from human-machine interface research. This system is currently under revision.

Inaccurate or incorrect mental models of how the system worked were apparent at the general level as well. For example, very young subjects entered queries in sentence or phrase form - they tried to conduct a natural language dialog with the system. The present system clearly cannot compensate for user's mental models that ascribe intelligence to it. Having little experience with encyclopedias of any kind, these young children modeled use of the computer system on their most common strategy for information-seeking - asking an "expert." Older children modeled the electronic encyclopedia on the familiar print versions.

Another common problem was subjects' poor mental models for searching in general. For example, some subjects added terms to queries that yielded no hits and contained ANDed terms. The system could trap such logical errors by pointing out that adding additional terms actually narrows a query (more terms yield fewer hits). Such forms of automatic help and remediation promote accurate mental model development, but if applied too often they may deter beginners from accomplishing immediate tasks and thus discourage continued use.

Perhaps an incremental approach is possible with systems that themselves record histories of use for users, but for hypertext systems like public access encyclopedias, we must find a balance between support for Boolean connectives and automatic adjustment of default conditions. For such systems, we believe that we can achieve significant cognitive advantage by amplifying low cognitive load browsing strategies and making the high cognitive load strategies that require Boolean manipulations and default adjustments transparent to the novice user.

The Electronic Encyclopedia is much more controlled than other hypertext systems in that jumps from article to article depend on a list of articles retrieved by a query. A user who wants to see an article not on the retrieved list must pose another query or enter a separate mode that allows lookup of single articles by title. On the other hand, the full-text search feature is totally under the control of the user. Problems of information overload did occur, but were minimized by the combined effects of the database (a set of generic, highly organized encyclopedia articles) and search rules (easy modification of queries and easy use of the scan and select browsing strategy). Complex, highly specific, or loosely organized databases may require distinct designs.

\section{Design issues}

Designers of hypertext systems or databases should consider the informationseeking framework (Figure 1). The over- 
all design must attend to the physical system, the conceptual model the system presents (the user interface), and the mental model the user is expected to develop for the system. Design decisions that affect information-seeking in hypertext systems are related to defining access points, creating the user interface, and providing search strategy features. These decisions interact in unexpected ways, but thoughtful designs can preserve power and flexibility while reducing complexity of use.

Defining access points. In traditional electronic databases, access points for retrieval include characters, fields, records, and tables or files. Access points may be restricted to selected points, organized in alphabetical or hierarchical sequence, or multiply indexed. Conside the access points typically available in printed books: table of contents, indexes (author, subject, permuted, etc.), glossary, chapter, article (section), physical page, paragraph, footnotes, reference notes, lists, and appendices. Pagination is critical to access in books, since page number is the primary pointer to a field location.

Hypertext databases can support all these access points except physical page. A screenful of text cannot be used as an analog of the physical page in systems that permit control of text window size, scrolling, and stacking or juxtaposing windows However, since the machine can assist in maintaining pointer information, hypertext systems can link field locations in many other ways, including electronic bookmarks, temporary notepads, graphic maps, backward citation pointers, and pattern matches on characters or words.

Hypertext systems also allow other access points that books do not, such as cumulative record of path, string search, animation, sound, video, or immediate link to related software. (For example, access software for Microsoft's Bookshelf on CD-ROM can be memory resident for immediate use from within another program.) Whether users can take advantage of these features remains to be determined.

A key decision for designers or authors is what access points to define and how to link these points. Users will likely expect access points at least as rich as those available in books. Designers must decide how much more to offer and how much unrestrained jumping from point to point to allow. Links at every word to every word are clearly not desirable from the perspective of user or system performance. The

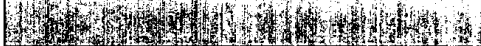

Since the organization of the information in hypertext is not linear, mechanisms for selection and feedback are critical to good design.

trade-offs in machine overhead and user cognitive load (in the form of overchoice) must be weighed carefully. Designers should consider the targeted task domains and typical user population in deciding how fine the access points should be and what links among access points should be visible to users.

Creating the user interface. An interface enables users to perform their tasks by providing selection mechanisms, feedback mechanisms, and input/output devices. In a book the default sequence is top-down due to the linear nature of the text. The interactive and flexible characteristics of hypertext require users to make more choices (selections) in searching for information. Furthermore, each selection requires appropriate and understandable feedback to maintain a fruitful interaction. Since the organization of the information in hypertext is not linear, mechanisms for selection and feedback are critical to good design.

Results from menu design research ${ }^{8}$ offer some guidance, but there are tradeoffs to consider. The key issue is one of user control. The extreme cases range from allowing the user to jump anywhere from anywhere (hyperchaos) to forcing the user through a linear sequence of screens with no deviation possible (drill and practice). The degree of user control provided will depend on the user, his or her purpose, and the task domain. Expert users who are specialists in the task domain will welcome great power and control, but novices to the system and the task domain will likely benefit from limited menus and less control.

The direct manipulation approach with embedded menus seems very ef fective for hypertext applications, but there are many variations on the theme. We believe that embedded menus provide meaningful task domain (as opposed to computer domain) terms and concepts, thereby reducing disorientation. However, we need more research to help guide designers in choosing highlighting techniques to indicate selectable text or graphic items without distracting too much from the content.

Similarly, feedback can range from cryptic codes to pseudo-intelligent contextsensitive help. Experts will quickly seek ways to avoid lengthy feedback because it slows down the dialog and impedes progress toward their goals. On the other hand, too little feedback will frustrate and confuse novices, yet too much can distract them. One approach for hypertext systems in public access settings permits users to specify their level of knowledge of the database content, information-seeking experience and purpose, and previous experience with this and other systems. Feedback settings can then be adjusted accordingly, with the user able to change settings at any point in a session.

Input/output devices affect user information-seeking performance at the behavioral level. Good choices on the part of the system designer can facilitate ease and efficiency of use, but poor choices can lead to user frustration or fatigue. Pointing devices such as touchscreen, mouse, jump-arrow keys, or keyboards need to be refined and evaluated. Improvements in screen readability through proper font design, text/background color pairs, or higher resolution would benefit users. Increased screen size and multiple window strategies also require investigation. Mapping the proper input/output devices to the system requires consideration of user, setting, and task domain characteristics.

Setting default conditions for these and other issues will depend on how the designer views the typical user. A range of alternative selection mechanisms can then be provided for use by atypical users. Mixed strategies are also possible. For example, a linear "tour" could be threaded through a complex network to offer new users a guided introduction.

Providing search strategy features. Search features like Boolean connectives, string search, proximity limits, scope limits, and truncation facilitate rapid access to information, but cause additional cognitive load on the part of the user and substantial preprocessing of the database itself. Systems that provide only browsing features allow casual, low cog- 
nitive load exploration, but are typically inefficient for directed search tasks or fact retrieval. Defining a hybrid system that guides discovery seems an appropriate compromise, but involves a number of trade-off decisions. How deeply the database is indexed, whether some automatic controlled vocabulary is included, and how feedback is summarized and even formatted on the screen affect the strategies users will apply. If every word is indexed, the possibility of information overload increases. Therefore, features for filtering such as frequency of occurrence per node or support for NOT operators must be enhanced. If a controlled vocabulary is included, automatic thresholds must be established, or the user must be prompted to apply the controlled vocabulary or be alerted to its effects. For example, in an encyclopedia, a query that retrieved more than 50 articles could automatically trigger a narrowing function.

Secondary databases (containing pointer information) such as on-line catalogs or bibliographic databases and highly structured primary databases require analytical strategy support. Text or graphic databases seem to invite scan and select browsing strategies, although the size and complexity may warrant some additional indexing to improve user search efficiency. In general, each feature added to a system demands additional machine overhead. Many also add user cognitive load. These effects must be considered in all design decisions.

The flexibility/complexity trade-off. Systems transparent to one user may frustrate and impede others. Flexibility inevitably leads to complexity. Just as printed indexes and directories are organized to facilitate retrieval (nobody reads the phone book), so electronic information systems must be organized to suit the typical purposes of anticipated users All designers must grapple with the issue of when to stop adding features-they face a law of diminishing returns. Empirical results and the marketplace will determine what the next version of a system should include and how much complexity users can tolerate.

A similar design issue is the tension between the learnability and applicability of a system. A system that is easy to learn may not be easy to apply in full. Results from cognitive science demonstrate that users' mental models will depend on initial training or experience with a system. An incomplete and simple conceptual model

January 1988

\begin{tabular}{c}
\hline \\
In general, each \\
feature added to a \\
system demands \\
additional machine \\
overhead. Many also \\
add user cognitive \\
load.
\end{tabular}

used to present the system to new users may limit their understanding of the system and their ability to apply it to future problems.

One approach to this problem, used in systems like Hypercard, is to choose a familiar metaphor (stacks of cards) and provide several levels of application (user preferences), examples, and help. The metaphor of the familiar flat index card will surely facilitate initial learnability, but may limit applicability of the multidimensional electronic card. As information systems continue to increase in complexity and power, they will likely become more difficult to learn and apply.

Some designers believe in adaptive systems, in which the computer is programmed to recognize user skills or information needs and then modify the interface or guide the user to the desired destination. Another school concentrates on adaptable systems in which the user is given the power to alter the user interface or is offered a rich variety of traversal methods. Adaptive systems represent an attractive but unproven idea, while adaptable systems place a greater burden on the user even as they provide increased control.

Hypertext systems offer the potential for highly personalized informationseeking if designers can apply principles from traditional information systems and the results of empirical studies.

I nevitably, the application of computers as cognitive augmenting agents will improve cognitive performance and change the way we think. An empirical base of evidence is coalescing around research and development on how to create hypertext systems, how to write using hypertext systems, how to read using hypertext systems, and how to find information in hypertext systems.

Our experience suggests that a general information-seeking framework that includes setting, task domain, user, search system, and outcomes aids the design and study of hypertext. Key design issues include

- finding the correct information unit granularity for particular task domains and users;

- presenting interfaces with low cognitive load for selection and feedback mechanisms, and reasonable default conditions; and

- striking a balance between analytical and browsing search strategies.

The general problem of maximizing power and flexibility while minimizing complexity of use must always be attacked.

Although much remains to be learned about how users apply hypertext for information-seeking, clearly these systems offer distinct advantages for finding facts, browsing knowledge, and-we hopeacquiring wisdom. $\square$

\section{References}

1. T.H. Nelson, Literary Machines, Swarthmore, Penn., 1981. Available from Nelson.

2. D. Engelbart, "A Conceptual Framework for Augmentation of Man's Intellect," in Vistas in Information Handling, Vol. I, Spartan Books, Washington, D.C., 1963, pp. 1-29.

3. J. Conklin, "Hypertext: An Introduction and Survey," Computer, Sept. 1987, pp. 17-41.

4. N. Yankelovich, N. Meyrowitz, and A. van Dam, "Reading and Writing the Electronic Book," Computer, Oct. 1985, pp. 15-30.

5. F. Halasz, T. Moran, and R. Trigg, "Notecards in a Nutshell," CHI+ GIConf. Proc.: Human Factors in Computing Systems and Graphics Interfaces, 1987, pp. 45-52.

6. D. Goodman, "The Two Faces of HyperCard," Macworld, Oct. 1987, pp. 122-129.

7. R. Marcus, "An Experimental Comparison of the Effectiveness of Computers and Humans as Search Intermediaries," $J . A m$. Society for Information Science, Vol. 34, 1983, pp. 381- 404.

8. B. Shneiderman, Designing the User Interface: Strategies for Effective Human Computer Interaction, Addison-Wesley, Reading, Mass., 1987. 


\section{STRUCTURED DEVELOPMENT FORUM $X$}

AUGUST 8th-11th, 1988

SAN FRANCISCO, CALIFORNIA

\section{CALL FOR}

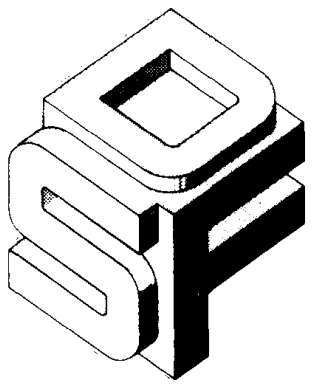

\section{PARTICIPATION}

The Structured Development Forum (SDF) is a conference devoted to presenting and promoting improved methods and automation for the development of software based systems. SDF members represent a broad hase of system developers (business, dat base, en pineering, real-time and other systems). Members also include leaders in automated todls and me hods consultants/trainers. SDF is sponsotel by me hber companies.

TECHNI AL PAPES $S$ will concentrate on technical advandes and proje t experience in the application of methods hools and Send 5 copies of 50 structured systems development. LLNL, PQ Box BO word abstract to Dr. Lin Zucconi,

March 988.

TUTORI LS will $f$ cus on innovative new methods and concepts. Sen 5 copies of detailed outline to $\mathrm{Mr}$. Joe Brandt, LLNL, O. Box 808, L-438, Livermore, CA 94550, by March 1 / 988.

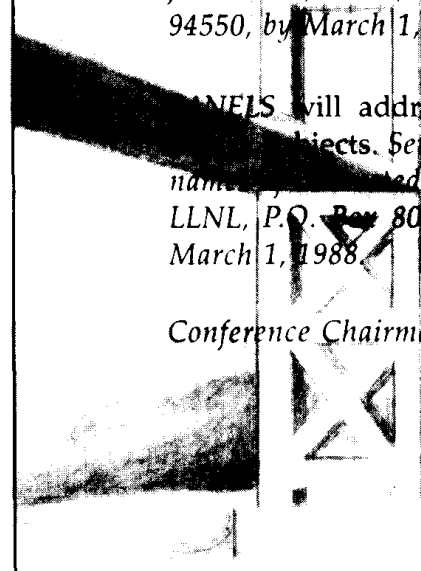

Is current and controversial in5 copies of 500 toord abstract and Qanelists to Ms. Barbara Campbell. LLNL, P.P. 80 80, 25, Livermore, CA 94550, by 11
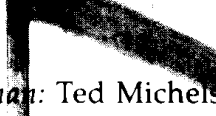

Ted Mich
LLNL P.O. Box 808 L-308

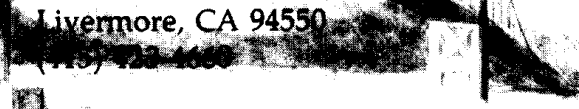

A.

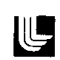

Sponsored by Lawrence Livermore National Laboratory
9. G. Gentner and A. Stevens, eds., Mental Models, Lawrence Erlbaum Associates, Hillsdale, N.J., 1983.

10. C.L. Borgman, "The User's Mental Model of an Information Retrieval System: An Experiment on a Prototype On-line Catalog,"' Int'l J. Man-Machine Studies, Vol. 24, 1986, pp. 47-64.

11. W. Zoellick, "CD-ROM Software Development," Byte, Vol. 11, 1986, pp. 177-188.

12. B. Shneiderman, "User Interface Design and Evaluation for an Electronic Encyclopedia," in Cognitive Engineering in the Design of Human-Computer Interaction and Expert Systems, G. Salvendy, ed., Elsevier, Amsterdam, 1987, pp. 207-223.

13. G. Marchionini, "Information-Seeking Strategies of Novices Using a Full-Text Electronic Encyclopedia,' J. Am. Society for Information Science, in press.

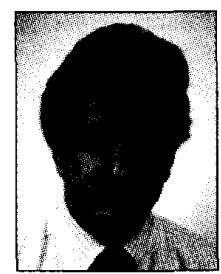

Gary Marchionini is an assistant professor in the College of Library and Information Services at the University of Maryland. His research interests center on information seeking in electronic environments and supporting casual users of full-text databases.

Marchionini received his PhD in mathematics education with an emphasis on educational computing from Wayne State University.

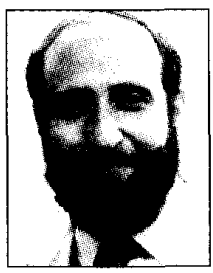

Ben Shneiderman is an associate professor in the Department of Computer Science, head of the Human-Computer Interaction Laboratory, and member of the Institute for Advanced Computer Studies, all at the University of Maryland. Shneiderman is on the editorial advisory board of the International Journal of ManMachine Studies and edits the Ablex Publishing $\mathrm{Co}$. book series on human-computer interaction.

Readers may write to Shneiderman at the Dept. of Computer Science, University of Maryland, College Park, MD 20742.

COMPUTER 